\title{
Sidelobe Performance Analysis of Noise Waveforms Considering the Doppler Mismatch
}

\author{
Gabriel Beltrão \\ SnT \\ University of Luxembourg \\ Luxembourg \\ gabriel.tedgue-beltrao@uni.lu \\ Leandro Pralon \\ Radar Division \\ Brazilian Army Technology Center \\ Rio de Janeiro, Brazil \\ pralon.leandro@eb.mil.br
}

\author{
Mohammad Alaee-Kerahroodi Bhavani Shankar M.R. \\ $\operatorname{SnT}$ \\ SnT \\ University of Luxembourg \\ Luxembourg \\ mohammad.alaee@uni.lu
}

\begin{abstract}
Waveform design and optimization algorithms generally assume a zero-Doppler ideal case to reach an optimum or satisfactory solution in terms of the matched filter output. Therefore, its performance is usually characterized only in terms of the resultant waveforms autocorrelation function, neglecting the practical situation in which the received signal is modulated by the target's Doppler shift. Within this context, this work investigates the Doppler mismatch effects in the Integrated Sidelobe Level (ISL) performance of previously designed/optimized noise waveforms. The analysis has shown that, despite much better results for steady targets, the increasing Doppler mismatch reduces the ISL performance of optimized waveforms, until similar levels achieved when no optimization is performed. To address that, a subpulse Doppler processing approach is also considered, and the results have shown that, besides increasing the Doppler tolerance, it has also increased the optimized waveform robustness to the Doppler mismatch, reducing the resultant ISL loss and thus extending its applicability.
\end{abstract}

Index Terms - noise radar, ambiguity function, sidelobes, subpulse processing.

\section{INTRODUCTION}

The stochastic nature of noise waveforms make them intuitively attractive for applications that require high performance against external interferences and low-probability-of-intercept (LPI) [1]. Additionally, good range and Doppler resolution, low sidelobes and high Doppler tolerance can also be achieved if properly taken into consideration during the waveform design process [2]. Several applications of noise radars have been considered so far, including Ultrawideband [3], Throughthe-Wall [4] and weather monitoring [5], just to cite a few.

The design and optimization of specific features is one of the key techniques for improving the performance of modern radar systems and have received considerable attention during the last few years [6], [7]. Several procedures specifically designed for random waveforms have been recently proposed and evaluated [8]-[10]. However, the resultant performance is usually addressed only in terms of the waveform autocorrelation function, which neglects the practical situation in which the received signal is modulated by the target's Doppler shift and, therefore, a mismatch with the matched filter's reference function is expected. Considering noise waveform

This work was supported by the National Research Fund of Luxembourg, under the AFR Industrial Fellowship Grant for Ph.D. projects (reference 14269859). systems, this analysis is even more important as range/Doppler residues of the cross-ambiguity function often lead to masking problems between strong and weak targets [11].

In this paper, we investigate the Doppler mismatch effects in the Integrated Sidelobe Level (ISL) performance of previously designed/optimized noise waveforms. Additionally, the subpulse Doppler processing proposed in [12] is also considered, and its sidelobe performance is compared to the conventional Doppler processing.

\section{Problem Formulation}

The proper characterization of a radar's waveform must comprise the derivation of its ambiguity function, which is a two dimensional function (time delay and Doppler frequency) describing the output of the matched filter as a function of a presumable Doppler shift caused by the target's radial velocity [13].

When considering noise waveforms, the transmitted signal's complex envelope in a given $i$ th interval, $\tilde{s}_{i}(t)$, is a sample function of a complex stationary stochastic process $\tilde{s}(t)$. As a consequence, the complex envelopes of the received signals and the corresponding matched filter outputs are all characterized by complex stationary random processes. The expected value of the narrow-band ambiguity function can then be written as [14]

$$
E\left[\chi_{\tilde{s}}\left(\tau, f_{d}\right)\right]=E\left[\int_{-\infty}^{\infty} \tilde{s}(t) \tilde{s}^{*}(t-\tau) e^{j 2 \pi f_{d} t} d t\right],
$$

where $f_{d}$ is the Doppler frequency, $\tau$ is the time delay and $j$ is the imaginary unit.

In pulsed radars, the transmit signal is time limited with duration $\tau_{s}$, characteristic that is mathematically represented by the rectangular function rect $\tau_{s}(t)$, in order to assure that the process $\tilde{s}(t)$ remains stationary. Furthermore, assuming that $f_{d}$ is deterministic, the expectation in (1) is over the distribution of $\tilde{s}(t)$, therefore, (1) can be rewritten as

$E\left[\chi_{\tilde{s}}\left(\tau, f_{d}\right)\right]=\int_{-\infty}^{\infty} E\left[\tilde{s}(t) \tilde{s}^{*}(t-\tau)\right] \operatorname{rect}_{\tau_{s}}(t) e^{j 2 \pi f_{d} t} d t$,

Since $\tilde{s}(t)$ is stationary, (2) can be further simplified as

$$
E\left[\chi_{\tilde{s}}\left(\tau, f_{d}\right)\right]=R_{\tilde{s}}(\tau) \int_{-\infty}^{\infty} \operatorname{rect}_{\tau_{s}}(t) e^{j 2 \pi f_{d} t} d t,
$$


where $R_{\tilde{s}}(\tau)$ is the autocorrelation function of the stochastic process $\tilde{s}(t)$. Note that the integral in (3) is the Fourier transform of the rectangular pulse. Therefore, the expected value of the ambiguity function of random signals can be written as

$$
E\left[\chi_{\tilde{s}}\left(\tau, f_{d}\right)\right]=\tau_{s} R_{\tilde{s}}(\tau) \operatorname{sinc}\left(f_{d} \tau_{s}\right),
$$

which has a similar format as in [15]. When the Doppler shift is null, (4) reduces to the autocorrelation function of the transmitted signal (the designed waveform).

Considering now $\tilde{\mathbf{s}}=[\tilde{s}(1), \tilde{s}(2), \ldots, \tilde{s}(N)]^{T} \in \mathbb{C}^{N}$, a discrete realization of the transmitted signal $\tilde{s}(t)$, with $N$ being the number of samples in each pulse (code length). The aperiodic autocorrelation function associated with $\tilde{\mathbf{s}}$ can be written as

$$
R_{\tilde{s}}(k)=\sum_{i=1}^{N-k} \tilde{s}(i)^{*} \tilde{s}(i+k), \quad k=0, \ldots, N-1 .
$$

The ISL, also called noise floor, or residual fluctuation level, is commonly used to design and evaluate waveforms in terms of its autocorrelation properties [17], and it can be defined as

$$
\mathrm{ISL}=\sum_{k=1}^{N-1}\left|R_{\tilde{s}}(k)\right|^{2} .
$$

It can be seen that the transmitted signal autocorrelation function plays an important role in the performance of radar systems that employ random waveforms. In fact, for a given pulse duration, the expected ambiguity function can be shaped by the appropriate choice of the waveform autocorrelation. This conclusion points to the importance of obtaining (either by design or optimization) a narrow and bell-shaped (nearthumbtack) autocorrelation function, with low sidelobes.

Even though the ambiguity function first order moment in (4) can be considered a good indicator of the waveform's performance, to properly analyze the sidelobe levels in Noise Radar systems it is necessary to investigate its second order moment, given by

$$
E\left[\left|\chi_{\tilde{s}}\left(\tau, f_{d}\right)\right|^{2}\right]=E\left[\chi_{\tilde{s}}\left(\tau, f_{d}\right) \chi_{\tilde{s}}^{*}\left(\tau, f_{d}\right)\right]
$$

Expanding (7) yields

$$
\begin{aligned}
& E\left[\left|\chi_{\tilde{s}}\left(\tau, f_{d}\right)\right|^{2}\right]=\int_{-\infty}^{\infty} \int_{-\infty}^{\infty} E\left[\tilde{s}(\beta+\tau) \tilde{s}^{*}(\beta) e^{j 2 \pi f_{d} \beta}\right. \\
& \left.\cdot \operatorname{rect}_{\tau_{s}}(\beta) \tilde{s}^{*}(\alpha+\tau) \tilde{s}(\alpha) e^{-j 2 \pi f_{d} \alpha} \operatorname{rect}_{\tau_{s}}(\alpha)\right] d \alpha d \beta
\end{aligned}
$$

Assuming that the transmit signal is phase or frequency modulated, it is possible to write

$$
\begin{gathered}
E\left[\left|\chi_{\tilde{s}}\left(\tau, f_{d}\right)\right|^{2}\right]=\int_{-\infty}^{\infty} \int_{-\infty}^{\infty} E\left[e^{j z(\alpha, \beta)}\right] e^{-j 2 \pi f_{d} \alpha} \\
\cdot e^{j 2 \pi f_{d} \beta} \cdot \operatorname{rect}_{\tau_{s}}(\alpha) \cdot \operatorname{rect}_{\tau_{s}}(\beta) d \alpha d \beta
\end{gathered}
$$

with $z(\alpha, \beta)$ being the random variable defined as

$$
z(\alpha, \beta)=\theta(\beta+\tau)-\theta(\beta)-\theta(\alpha+\tau)+\theta(\alpha)
$$

When $\theta($.$) is a zero mean Gaussian random process, the$ random variable $z(\alpha, \beta)$ is also Gaussian with zero mean.
Hence, after some mathematical manipulations, it is possible to obtain

$$
\begin{aligned}
& E\left[\left|\chi_{\tilde{s}}\left(\tau, f_{d}\right)\right|^{2}\right]=4 P^{2} \tau_{s} \int_{-\infty}^{\infty} \operatorname{tri}\left(\gamma / \tau_{s}\right) \exp \left\{-2 R_{\theta}(0)+\right. \\
& \left.+2 R_{\theta}(\tau)+2 R_{\theta}(\gamma)-R_{\theta}(\gamma+\tau)-R_{\theta}(\gamma-\tau)\right\} e^{-j 2 \pi f_{d} \gamma} d \gamma
\end{aligned}
$$

where $\gamma=\alpha-\beta$ and $\operatorname{tri}\left(\gamma / \tau_{s}\right)$ is the triangular function with duration $2 \tau_{s}$, given by

$$
\operatorname{tri}\left(\gamma / \tau_{s}\right)=\left\{\begin{array}{lll}
1-\left|\gamma / \tau_{s}\right| & ; & |\gamma| \leq \tau_{s} \\
0 & ; & |\gamma|>\tau_{s}
\end{array}\right.
$$

Analogous to the previous analysis in [18], two regions can be identified in (11), that provide good approximations for both the main lobe as well as the stationary sidelobe level. First, note that, if $\tau$ is close to 0 , except for values of $\gamma$ close to zero, $2 R_{\theta}(\gamma) \approx R_{\theta}(\gamma+\tau)+R_{\theta}(\gamma-\tau)$, which leads to

$$
\begin{gathered}
E\left[\left|\chi_{\tilde{s}}\left(\tau, f_{d}\right)\right|^{2}\right]_{\mathrm{ML}}=4 P^{2} \tau_{s} \int_{-\infty}^{\infty} \operatorname{tri}\left(\gamma / \tau_{s}\right) \exp \left\{-2 R_{\theta}(0)+\right. \\
\left.+2 R_{\theta}(\tau)\right\} e^{-j 2 \pi f_{d} \gamma} d \gamma
\end{gathered}
$$

The integral in (13) becomes the Fourier transform of the aforementioned triangle function and hence

$$
E\left[\left|\chi_{\tilde{s}}\left(\tau, f_{d}\right)\right|^{2}\right]_{\mathrm{ML}}=\tau_{s}{ }^{2} R_{s}^{2}(\tau) \operatorname{sinc}^{2}\left(f_{d} \tau_{s}\right)
$$

We refer to this result as the main lobe characterization. Now, let us address the sidelobes levels characterization, i.e. the behavior of what can be considered an upper bound to the sidelobes levels [19], [20]. Note that, for large values of $\tau$, (11) presents a stationary behavior and, after some mathematical manipulations, it can be shown that the integrated sidelobe level is independent of $\tau$ and given by

$E\left[\left|\chi_{\tilde{s}}\left(\tau, f_{d}\right)\right|^{2}\right]_{\mathrm{SL}}=\tau_{s} \int_{-\infty}^{\infty} \operatorname{tri}\left(\gamma / \tau_{s}\right) R_{s}^{2}(\gamma) e^{-j 2 \pi f_{d} \gamma} d \gamma$

When no optimization is employed, the matched filter output power quickly converges to (15), which then dictates the ISL. Note that (15) is the Fourier transform of a product between two functions, which results in the convolution of their individual Fourier transform as

$$
E\left[\left|\chi_{\tilde{s}}\left(\tau, f_{d}\right)\right|^{2}\right]_{\mathrm{SL}}=\left.\tau_{s} \mathcal{F}\left[R_{s}^{2}\right]\right|_{f_{d}} * \operatorname{sinc}^{2}\left(f_{d} \tau_{s}\right)
$$

where $*$ and $\mathcal{F}[\cdot]$ are the convolution and Fourier transform operator, respectively. Since the Fourier transform of the squared autocorrelation function as a function of $f_{d}$ has a much slower decay than $\operatorname{sinc}^{2}\left(f_{d} \tau_{s}\right),(16)$ approaches

$$
\left.E\left[\left|\chi_{\tilde{s}}\left(\tau, f_{d}\right)\right|^{2}\right]_{\mathrm{SL}} \approx \tau_{s} \pi \mathcal{F}\left[R_{s}^{2}\right]\right|_{f_{d}=0}
$$

Therefore, under the assumptions made, the Doppler shift does not influence the stationary sidelobe level at the matched filter output when random phase or frequency modulated waveforms are employed. Nevertheless, in many radar applications, the sidelobe levels achieved in (17) can be too high, possibly masking other targets and thus leading to the necessity in optimizing the modulating signal in order to obtain lower sidelobe levels at the output of the matched filter. 
Fig. 1 shows, as examples, the ambiguity function of sequences designed according to [18] and [21]. In [18], a Gaussian random modulating process is used to design frequency modulated noise waveforms (FM noise) with Gaussian-like Power Spectral Density (PSD) and autocorrelation function. In [21], a Coordinate-Descent (CD) framework is proposed to design and optimize the ISL of random phase sequences. In Fig. 1.(a), a purely random (neither design nor optimization) noise waveform is also shown for reference. All considered cases are constant-modulus with bandwidth $B_{\tilde{s}}=10 \mathrm{MHz}$, duration $\tau_{s}=20 \mu$ s and size $N=200$ samples. While the FM noise waveform in 1.(b) presents a reasonable amount of sidelobes, as a result of the optimization process, it can be seen in 1.(c) that the sidelobes of the ISL-optimized waveform are greatly reduced. All of them present very low Dopplertolerances as a result of the near-thumbtack mainlobe.

\section{A. Subpulse Doppler Processing}

In [12], a subpulse processing architecture was proposed to reduce the Doppler mismatch loss of long range noise radar systems. It can be briefly described as a set of subpulse matched filters followed by a 2D-DFT, and its Doppler estimation performance has already been addressed in [22]. Since the sidelobe behavior of the aforementioned waveforms is influenced by the pulse duration $\tau_{s}$, the referred approach is revisited for the investigation of its sidelobe performance considering Doppler mismatched scenarios. Fig. 2 shows the proposed approach block diagram.

The first step in the procedure is to divide the $i^{t h}$ reference signal, into $m$ subpulses. Then, $m$ matched filter operations are performed, considering the received signal and each reference subpulse $k$. Analogously to (4), the expected value of each output is given by

$$
\begin{aligned}
& E\left[\tilde{y}_{i k}(t)\right]= \\
& \quad \frac{\tau_{s}}{m} A R_{\tilde{s}}\left(t-T_{0}-T_{i k}\right) \operatorname{sinc}\left(f_{d} \frac{\tau_{s}}{m}\right) e^{-j 4 \pi \frac{\left(R_{0}+v T_{i k}\right)}{\lambda}}
\end{aligned}
$$

where $[i, k]$ represents a given [pulse, subpulse] set, $t$ is the fast time, $T_{0}=2 R_{0} / c$, with $c$ being the speed of light and $R_{0}$ the target's radial distance, $v$ is the target's velocity, $\lambda$ is the transmit signal's wavelength and $T_{i k}=\tau_{s} \frac{k}{m}+(i-1)$ PRT, with PRT being the pulse repetition interval.

Assuming that $T_{i k}$ is small enough not to induce target range migration, the peak positions can be assumed to be the same, occurring at $t=T_{0}$, for all the $m$ outputs. A DFT stage is then performed within the subpulse dimension, $k$, characterizing a coherent integration operation, which compensates the $1 / \mathrm{m}$ factor in (18). The output of this processing block, considering $t=T_{0}$, can be shown to be, for $f \in\left[\frac{-m}{2 \tau_{s}}, \frac{m}{2 \tau_{s}}\right)$,

$$
\left.E\left[\tilde{Y}_{i}^{\prime}(f)\right]\right|_{t=T_{0}}=\tau_{s} A R_{\tilde{s}}(0) \operatorname{sinc}\left(f_{d} \frac{\tau_{s}}{m}\right) e^{-j 4 \pi \frac{\left(R_{0}+v T_{i}\right)}{\lambda}} \psi\left(f-f_{d}\right)(19
$$

where $T_{i}=(i-1) \mathrm{PRT}$, and $\psi(x)$ is the Aliased sinc function [13]. The last step of the subpulse approach is the DFTs in the pulse dimension, $i$, the same as the conventional Doppler processing, characterizing a final coherent integration stage. In

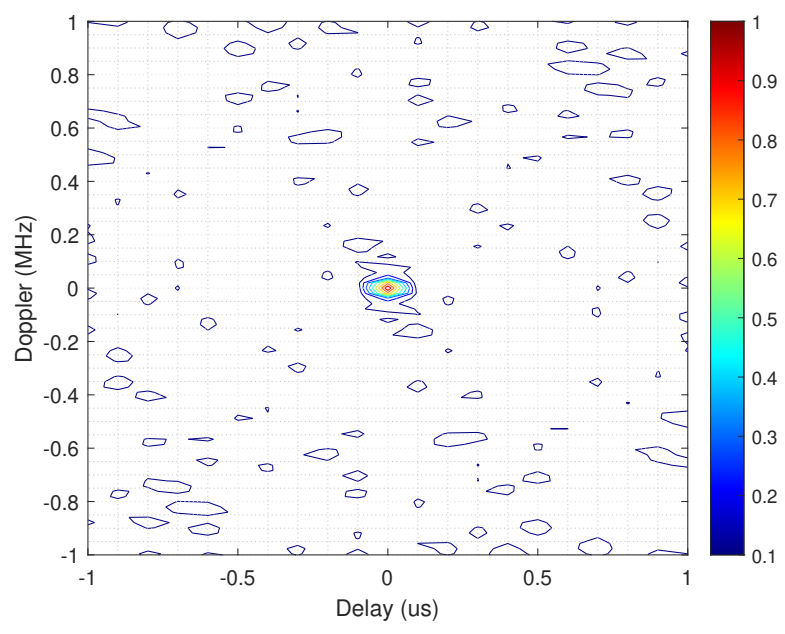

(a) Purely random noise waveform.

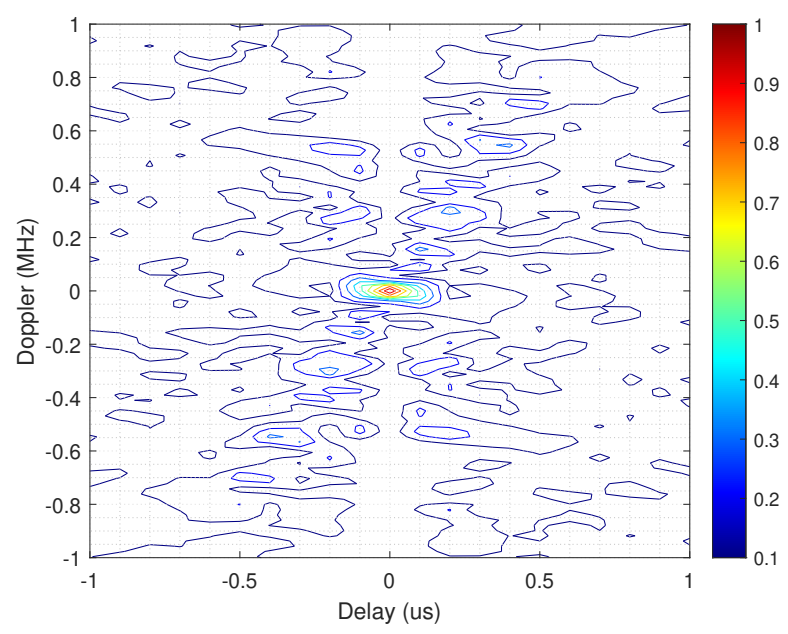

(b) Random FM noise waveform [18].

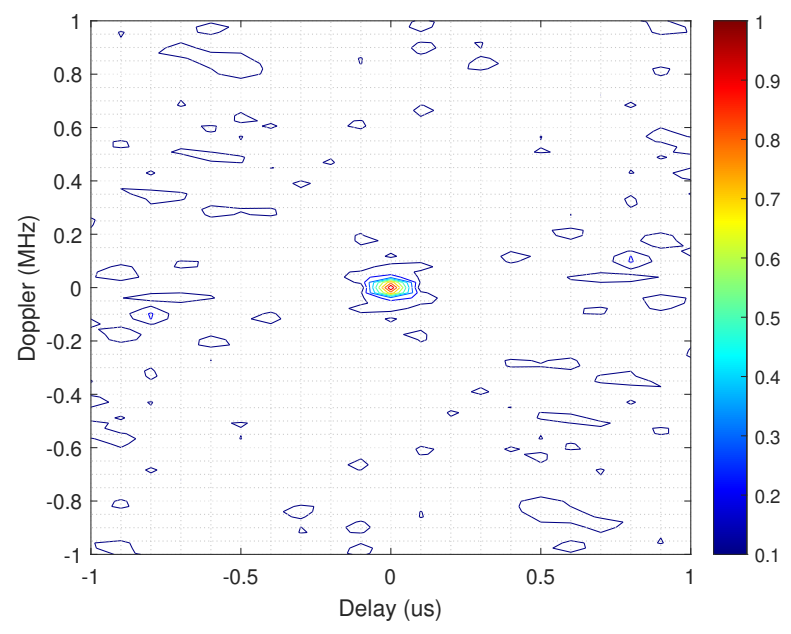

(c) ISL-optimized noise waveform [21].

Fig. 1. Ambiguity function of noise waveforms. 


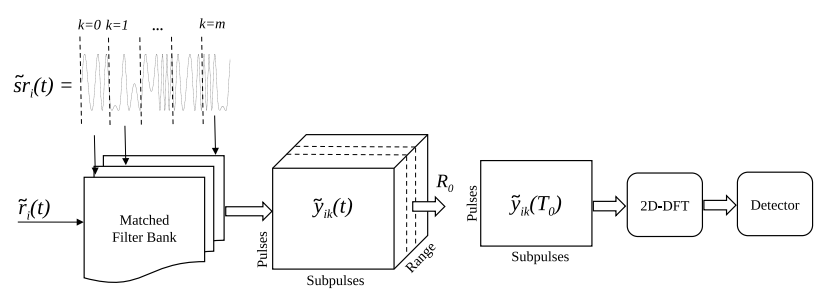

Fig. 2. Block diagram of the subpulse Doppler processing [12]

Fig. 2, the two DFTs are coupled into a single two-dimensional DFT operation over the Doppler map (pulse/subpulse) of each range bin.

\section{Performance Analysis}

In the present section, the sidelobe performance of the aforementioned ISL-optimized and FM noise waveforms are addressed, considering an increasing Doppler mismatch as the target's radial velocity increases accordingly. In order to take into consideration the effects of the signal processing chain, the ISL values are being calculated over the final target range profile, after the range matched filter and the conventional Doppler processing, i.e. the Discrete Fourier Transform (DFT) over the pulse dimension, for each range bin. Both waveforms are constant-modulus sequences, the pulse bandwidth is $B_{\tilde{s}}=5 \mathrm{MHz}$, with duration $\tau_{s}=100 \mu \mathrm{s}$ and size of $N=500$ samples. The final target's normalized power (received power after processing gain), in $t=T_{0}$, is also being presented, in order to provide a clear picture of the Doppler mismatch effect on the matched filter main lobe.

Fig. 3.(a) shows the final target's normalized power as a function of its velocity. As expected by the analysis in the previous Section, all the waveforms present the same behavior, with a strong attenuation as the target velocity increases, following the sinc-like pattern depicted in (14). It can be concluded that, independent of the design/optimization strategy, they all present similar (low) Doppler tolerances. The region between $450 \mathrm{~m} / \mathrm{s}$ and $550 \mathrm{~m} / \mathrm{s}$ does not provide reliable measurements as the peak is strongly attenuated to the same level of the sidelobes.

The calculated ISL values as a function of the target velocity can be seen in Fig. 3.(b). The pure random and the FM noise waveforms present a near-constant behavior, showing that their sidelobes are not affected by the target velocity, as explained by (16). The slight worse performance of the FM noise, in relation to the pure random waveform can be explained by its Gaussian-like autocorrelation function. Despite the superior performance of the ISL-optimized waveform up to $300 \mathrm{~m} / \mathrm{s}$ (an improvement of almost $20 \mathrm{~dB}$ for a zero-Doppler ideal target), for higher velocities, the mismatch caused by the target's Doppler shift increases the resultant sidelobes until a point in which its performance converges to the same levels as the non-optimized waveforms.

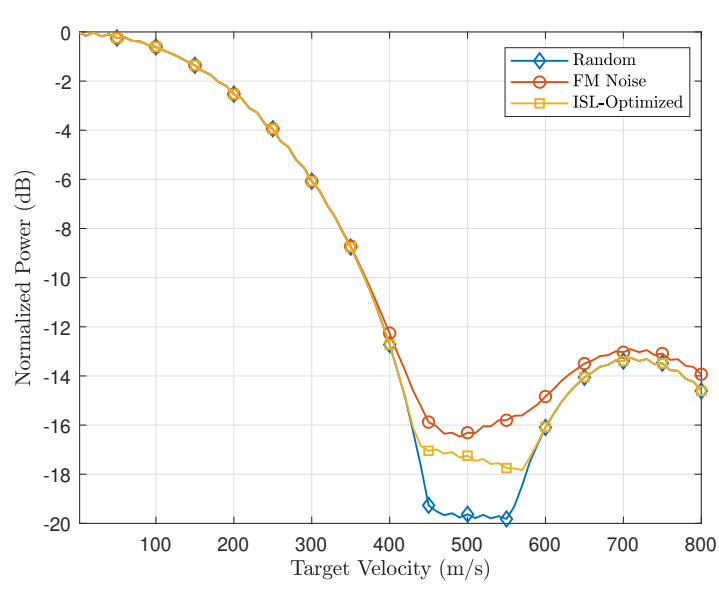

(a) Final targets's normalized power.

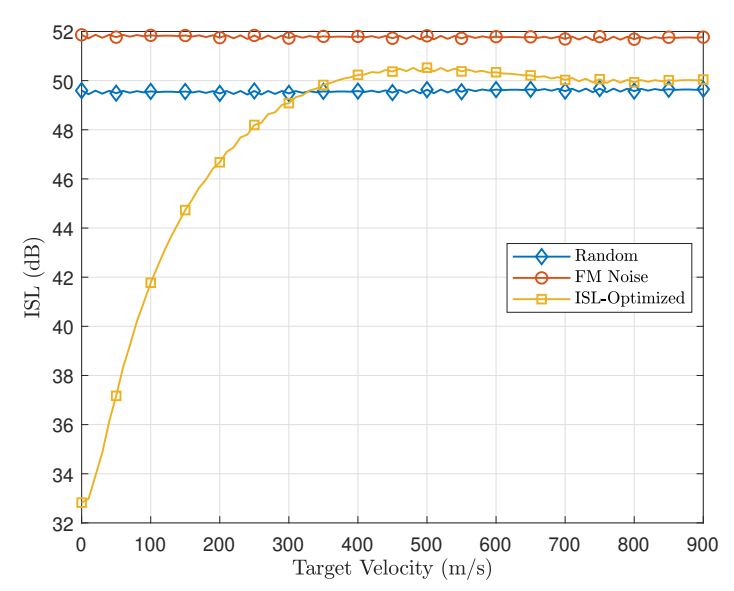

(b) Integrated Sidelobe Level (ISL).

Fig. 3. Mainlobe and sidelobe performance of noise waveforms, as a function of the target velocity, considering the conventional Doppler Processing.

Fig. 4 shows the obtained results when the conventional Doppler processing is replaced by the subpulse processing, with $m=4$ subpulses. It can be seen from Fig. 4.(a) that the Doppler tolerance has increased according to (18), not only for the FM noise waveform but also for the pure random and the ISL-optimized ones. The maximum losses were reduced from around $-16 \mathrm{~dB}$ to $-2.5 \mathrm{~dB}$. In terms of the ISL, the pure random and the FM noise presented again the nearly-constant behavior as in Fig. 4.(b), which shows that their sidelobe performance is not affected by the subpulse processing. On the other hand, the ISL-optimized waveform is still affected by the Doppler mismatch, but, in this case, the performance loss is greatly reduced by the subpulse approach, with a smoother variation over all velocities.

\section{Conclusions}

The sidelobe performance of some design/optimized noise waveforms, as a function of the Doppler mismatch, has been addressed, considering both the conventional and the subpulse Doppler processing. The ISL values have been calculated over 


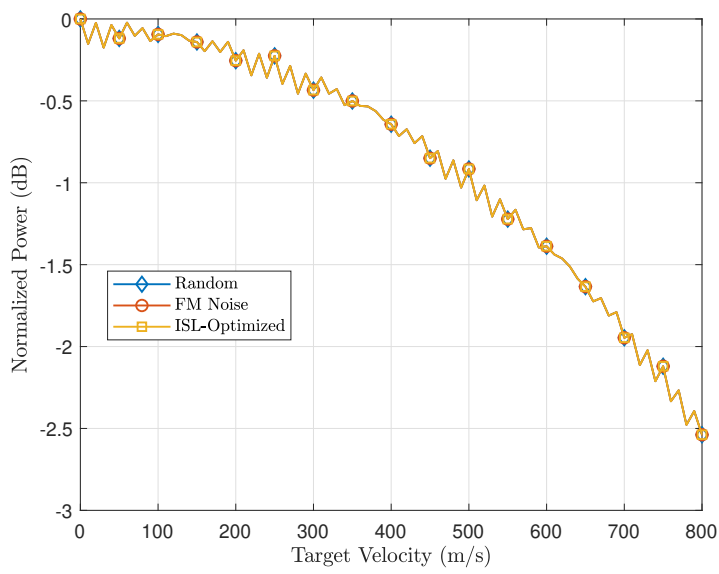

(a) Final targets's normalized power

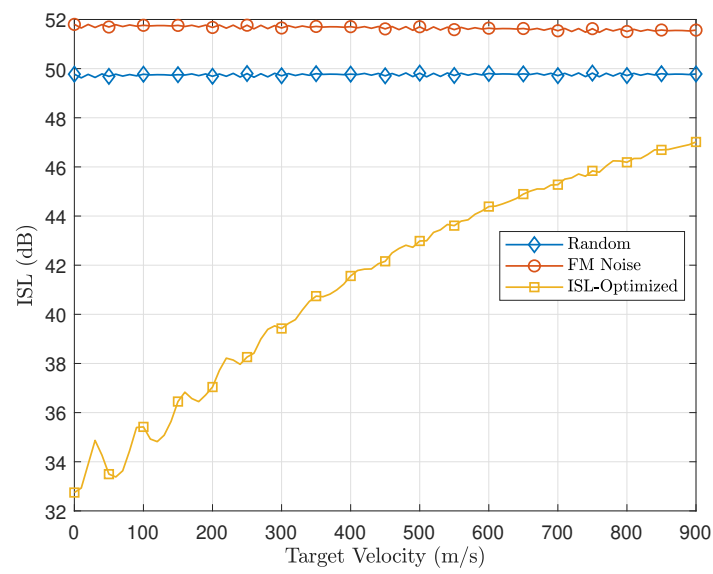

(b) Integrated Sidelobe Level (ISL).

Fig. 4. Mainlobe and sidelobe performance of noise waveforms, as a function of the target velocity, considering the subpulse Doppler Processing.

the target's final range profile, and presented as a function of the target's velocity. The target's final power has also been shown and compared to the corresponding ambiguity function expected value, as an indication of the Doppler tolerance of the analyzed waveforms.

The results have shown that, in terms of sidelobes, the Doppler mismatch has a low effect on the pure random and the FM noise waveforms, which presented a nearly-constant behavior over all velocities. On the other side, despite much better results for steady (zero-Doppler) and slow targets, due to the increased Doppler mismatch, the ISL performance of the optimized waveform was reduced until similar levels to the ones achieved when no optimization is performed. Additionally, It was also shown that, even after splitting the matched filter reference function into several subpulses, the subpulse Doppler processing not only increased the Doppler tolerance of the optimized waveform, but also its robustness to the Doppler mismatch, reducing the resultant ISL loss and thus extending its applicability.

\section{REFERENCES}

[1] G. Galati, G. Pavan, F. De Palo, and A. Stove, "Potential applications of noise radar technology and related waveform diversity," Proceedings International Radar Symposium, vol. 2016-June, no. i, pp. 1-5, 2016.

[2] T. Thayaparan, C. Wernik, R. Defence, and D. C. Ottawa, "Noise Radar Technology Basics," Security, no. December, 2006.

[3] Z. Li and R. M. Narayanan, "Doppler visibility of coherent ultrawideband random noise radar systems," IEEE Transactions on Aerospace and Electronic Systems, vol. 42, no. 3, pp. 904-915, 2006.

[4] H. Wang, R. M. Narayanan, and Z. O. Zhou, "Through-wall imaging of moving targets using UWB random noise radar," IEEE Antennas and Wireless Propagation Letters, vol. 8, pp. 802-805, 2009.

[5] L. Maslikowski, K. Kulpa, D. Glushko, and F. Yanovsky, "Atmospheric precipitation sensing with a short-range C-band noise radar," Proceedings International Radar Symposium, vol. 2, pp. 791-798, 2013.

[6] J. Song, P. Babu, and D. P. Palomar, "Sequence design to minimize the weighted integrated and peak sidelobe levels," IEEE Transactions on Signal Processing, vol. 64, no. 8, pp. 2051-2064, 2016.

[7] H. He, P. Stoica, and J. Li, "Designing unimodular sequence sets with good correlations - Including an application to MIMO radar," IEEE Transactions on Signal Processing, vol. 57, no. 11, pp. 4391-4405, 2009.

[8] J. S. Kulpa, L. Maslikowski, and M. Malanowski, "Filter-Based Design of Noise Radar Waveform with Reduced Sidelobes," IEEE Transactions on Aerospace and Electronic Systems, vol. 53, no. 2, pp. 816-825, 2017.

[9] C. A. Mohr, P. M. McCormick, and S. D. Blunt, "Optimized complementary waveform subsets within an FM noise radar CPI," 2018 IEEE Radar Conference, RadarConf 2018, pp. 687-692, 2018.

[10] M. Alaee-Kerahroodi, S. Sedighi, M. R. Bhavani Shankar, and B. Ottersten, "Designing (In)finite-alphabet Sequences via Shaping the Radar Ambiguity Function," ICASSP, IEEE International Conference on Acoustics, Speech and Signal Processing - Proceedings, vol. 2019-May, pp. 4295-4299, 2019.

[11] K. Kulpa, Signal Processing in Noise Waveform Radar. Artech House, 2013.

[12] G. Beltrao, L. Pralon, M. Menezes, P. Vyplavin, B. Pompeo, and M. Pralon, "Subpulse processing for long range surveillance noise radars," in International Conference on Radar Systems (Radar 2017). IET, 2017.

[13] M. A. Richards, Fundamentals of Radar Signal Processing. McGrawHill, 2014.

[14] N. Levanon and E. Mozeson, Radar Signals. Wiley-IEEE Press, 2004.

[15] J. M. Swiger, "Properties of the Periodic Ambiguity Function," IEEE Transactions on Aerospace and Electronic Systems, vol. 30, no. 3, pp. 938-941, 1994.

[16] S. Imani, M. M. Nayebi, and S. A. Ghorashi, "Colocated MIMO Radar SINR Maximization under ISL and PSL Constraints," IEEE Signal Processing Letters, vol. 25, no. 3, pp. 422-426, 2018.

[17] N. Levanon, "Radar principles," Wiley-Interscience, 1988

[18] L. Pralon, B. Pompeo, G. Beltrao, H. Cioqueta, B. Cosenza, and J. M. Fortes, "Random phase/frequency modulated waveforms for noise radar systems considering phase shift," 9th European Radar Conference, EURAD 2012, no. 2, pp. 314-317, 2012.

[19] F. Arlery, U. Tan, and O. Rabaste, "Generalization and improvement of the levenshtein lower bound for aperiodic correlation," IEEE Transactions on Information Theory, vol. 65, no. 2, pp. 1258-1266, 2018.

[20] L. Welch, "Lower bounds on the maximum cross correlation of signals (corresp.)," IEEE Transactions on Information theory, vol. 20, no. 3, pp. 397-399, 1974.

[21] M. A. Kerahroodi, A. Aubry, A. De Maio, M. M. Naghsh, and M. Modarres-Hashemi, "A coordinate-descent framework to design low PSL/ISL sequences," IEEE Transactions on Signal Processing, vol. 65, no. 22, pp. 5942-5956, 2017.

[22] F. Dar, S. S. Filho, G. Fraidenraich, M. D. Yacoub, M. Antonio, M. Miranda, and H. Cioqueta, "Probability of Detection for Unambiguous Doppler Frequencies in Pulsed Radars Using the Chinese Remainder Theorem and Subpulse Processing," 2019, pp. 138-142. 